\title{
Numerical simulations of the circumstellar medium of massive binaries
}

\author{
Allard Jan van Marle ${ }^{1}$ and Rony Keppens ${ }^{1,2,3}$ \\ ${ }^{1}$ Centre for Plasma Astrophysics, K.U. Leuven, \\ Celestijnenlaan 200B, B-3001, Heverlee, Belgium \\ email: [AllardJan.vanMarle] [Rony. Keppens] @wis. kuleuven . be \\ ${ }^{2}$ Sterrenkundig Instituut, University of Utrecht, \\ Postbus 80000, NL-3508TA, Utrecht, the Netherlands \\ ${ }^{3}$ FOM Institute for Plasma Physics Rijnhuizen, \\ P.O. Box 1207 NL-3430 BE Nieuwegein, the Netherlands
}

\begin{abstract}
We have made 3-D models of the collision of binary star winds and followed their interaction over multiple orbits. This allows us to explore how the wind-wind interaction shapes the circumstellar environment. Specifically, we can model the highly radiative shock that occurs where the winds collide. We find that the shell that is created at the collision front between the two winds can be highly unstable, depending on the characteristics of the stellar winds.
\end{abstract}

Keywords. methods: numerical, binaries: general, circumstellar matter, mass loss, stars: winds, outflows

\section{Introduction}

Massive stars emit powerful stellar winds that shape the environment around their progenitor stars. Recent advances in both hardware and software have made it possible to simulate the interactions of stellar winds emitted by binary stars. Such simulations were shown by Pittard (2009) and Pittard \& Parkin (2010) for close binaries. Here we add to these simulations by showing two wide orbit $(\mathrm{P}=1 \mathrm{yr})$ binaries. One for a Wolf-Rayet + O-star binary, the second an LBV + O-star binary.

\section{Astrophysical hydrodynamics for binary environments}

We use the MPI-AMRVAC code (Meliani et al. 2007) to solve the usual equations of hydrodynamics (conservation of mass, momentum) with the energy equation given by:

$$
\frac{\partial e}{\partial t}+\nabla \cdot(e \mathbf{v})+\nabla \cdot(p \mathbf{v})=-n^{2} \Lambda(T),
$$

with $\mathbf{v}$ the velocity, $p$ the pressure and $e$ the total energy density. The last equation includes the effect of radiative cooling $n^{2} \Lambda(T)$, which depends on local ion density, temperature and metallicity. The cooling curve $\Lambda(T)$ for gas at solar metallicity is obtained from Mellema \& Lundqvist (2002). For our binary simulations we use a Cartesian grid, centered around the centre of mass of the binary orbit. At each timestep we calculate

\begin{tabular}{lccccc}
\hline Type & Mass $\left[M_{\odot}\right]$ & $\dot{M}_{A}\left[M_{\odot} / y r\right]$ & $\dot{M}_{B}\left[M_{\odot} / y r\right]$ & $V_{A}[\mathrm{~km} / \mathrm{s}]$ & $V_{B}[\mathrm{~km} / \mathrm{s}]$ \\
\hline $\mathrm{LBV}+\mathrm{O}$ & $50+20$ & $1 \times 10^{-4}$ & $5 \times 10^{-7}$ & 200 & 2000 \\
$\mathrm{WR}+\mathrm{O}$ & $50+20$ & $5 \times 10^{-6}$ & $5 \times 10^{-7}$ & 1500 & 2000 \\
\hline
\end{tabular}



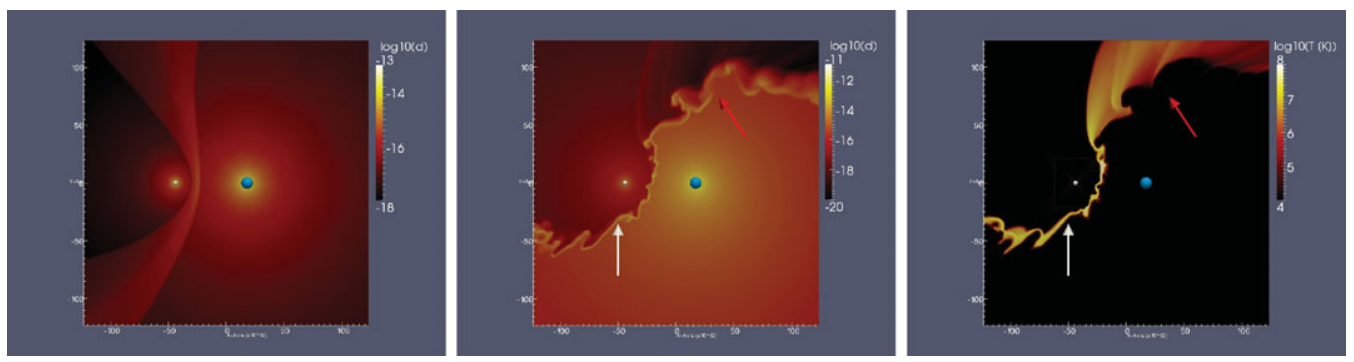

Figure 1. Circumstellar morphology for a Wolf-Rayet $+\mathrm{O}$ star binary (left) and the density (centre) and temperature (right) for a LBV+O star binary.

the location of each star and fill spheres, centered on these points, with free-streaming stellar wind material. The parameters for both simulations are given in the table below.

The density of the wind-shaped environment of a hydrogen rich Wolf-Rayet $+\mathrm{O}$ star binary, moving counter clockwise in orbit, after one complete orbit is shown in the lefthand image of fig. 1. The strong, high density Wolf-Rayet star (blue) wind clearly dominates. The interaction region is smooth and shows no sign of instabilities. This is due to the relatively ineffective radiative cooling. The shell formed by the colliding gas remains thick, preventing the formation of thin-shell instabilities.

The central and right hand plots of fig. 1 shows the circumstellar density and temperature respectively for a similar binary (same period and stellar masses) during a different evolutionary phase. The high mass star (blue symbol) now has a high density, relatively slow, Luminous Blue Variable (LBV) type wind. Due to the high density the radiative cooling allows the shell to be compressed leading to the formation of two types of thinshell instabilities. The type of instabilities depends on the nature of the shock. Between the stars and at the front of the bowshock (white arrow) the interaction is mostly radiative, so the thermalized zone is very thin. As a result the shell feels ram-pressure from both sides and forms non-linear thin-shell instabilities (Vishniac 1994). This effect is even more pronounced in the region exactly between the two stars, where the shock is strongest due to the head-on collision of the two winds, as was found in 2D simulations by Stevens et al. (1992). Further downstream (red arrow) the thermalized zone becomes quite thick. Therefore, the shell feels thermal pressure on one side and ram-pressure on the other, leading to the formation of linear thin-shell instabilities (Vishniac 1983).

\section{Acknowledgements}

A.J.v.M. acknowledges support from NSF grant AST-0507581, from the FWO, grant G.0277.08 and K.U.Leuven GOA/09/009.

\section{References}

Meliani, Z., Keppens, R., Casse, F., \& Giannios, D. 2007, mnras, 376, 1189

Mellema, G. \& Lundqvist, P. 2002, A\& $A, 394,901$

Pittard, J. M. 2009, mnras, 396, 1743

Pittard, J. M. \& Parkin, E. R. 2010, mnras, 403, 1657

Stevens, I. R., Blondin, J. M., \& Pollock, A. M. T.. 1992, ApJ, 386, 265

Townsend, R. H. D.. 2009, ApJS, 181, 391

Vishniac, E. T. 1983, ApJ, 274, 152

Vishniac, E. T. 1994, ApJ, 428, 186 